\title{
Person Re-id in Appearance Impaired Scenarios
}

\author{
Mengran Gou \\ http://www1.coe.neu.edu/ mengran/ \\ Xikang Zhang \\ zhangxk@ece.neu.edu \\ Angels Rates-Borras \\ ratesborras.a@husky.neu.edu \\ Sadjad Asghari-Esfeden \\ www1.coe.neu.edu/ sadjad/ \\ Octavia Camps \\ camps@coe.neu.edu \\ Mario Sznaier \\ msznaier@coe.neu.edu
}

\author{
Robust Systems Lab \\ Electrical and Computer Engineering \\ Northeastern University \\ Boston
}

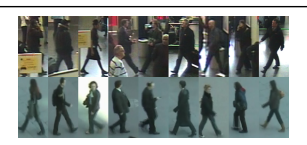

(a)

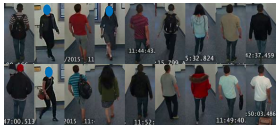

(b)

Figure 1: (a) Examples of persons wearing black suits; (b) Examples of images of the same person but wearing different clothing.

Person re-identification is critical in surveillance applications. Current approaches rely on appearance-based features extracted from a single or multiple shots of the target and candidate matches. These approaches are at a disadvantage when trying to distinguish between candidates dressed in similar colors (Figures 1(a)) or when targets change their clothing (Figures 1(b)). In this paper we propose a dynamics-based feature to overcome this limitation. The main contributions of this paper are: (i) A novel dynamics-based and Fisher vector encoded feature DynFV for re-id; (ii) Three new challenging "appearance impaired" datasets for re-id performance evaluation; and (iii) A comprehensive evaluation of the effect of choosing different spatio, spatio-temporal, and dynamics-based features on the performance of (unsupervised) re-id methods.

We propose to use soft-biometric characteristics provided by sets of dense, short trajectories (tracklets), which have been shown to carry useful invariants [1]. All tracklets are encoded by using pyramids of dense trajectories with Fisher vector encoding [2], as illustrated in Figure 2 and described in detail in the paper.

To illustrate the need for dynamic-based features we collected three challenging "appearance-impaired" datasets. Two of them consist of video sequences of people wearing black/dark clothing. They are subsets

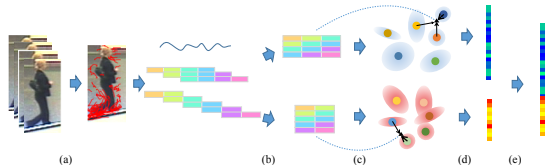

Figure 2: Pipeline of the proposed dynamics-based feature extraction.

of the iLIDS-VID and PRID 2011 datasets and we named them iLIDSVID BK and PRID 2011 BK, respectively. The third dataset, named the Train Station dataset (TSD), has sequences of persons with different clothing and accessories (Figure 1(b)). We compare unsupervised re-id performance when using different combinations of five different types of features. After combining LDFV and DynFV, the rank-1 accuracies have relative improvements of $142.1 \%$ on average for all three new datasets (Figure 3).

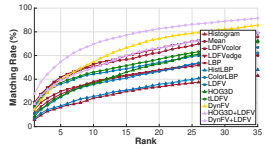

(a)iLIDSVID

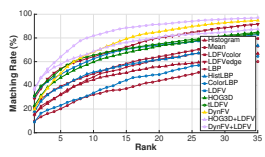

(c) PRID

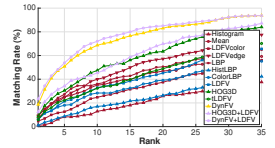

(b) iLIDSVID BK

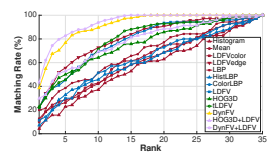

(d) PRID BK
Figure 3: CMC curves for iLIDSVID, PRID and the BK extension datasets

[1] B. Li, O. Camps, and M. Sznaier. Cross-view activity recognition using hankelets. In $C V P R$, pages 1362-1369, 2012.

[2] F. Perronnin, J. Sánchez, and T. Mensink. Improving the fisher kernel for large-scale image classification. In ECCV, pages 143-156. 2010. 